\title{
Modeling of the Radial Heat Flow and Cooling Processes in a Deep Ultraviolet $\mathrm{Cu}^{+} \mathrm{Ne}-\mathrm{CuBr}$ Laser
}

\author{
Iliycho Petkov Iliev, ${ }^{1}$ Snezhana Georgieva Gocheva-Ilieva, ${ }^{2}$ \\ Krassimir Angelov Temelkov, ${ }^{3}$ Nikolay Kirilov Vuchkov, ${ }^{3}$ \\ and Nikola Vassilev Sabotinov ${ }^{3}$ \\ ${ }^{1}$ Department of Physics, Technical University of Plovdiv, 25 Tzanko Djusstabanov Street, \\ 4000 Plovdiv, Bulgaria \\ ${ }^{2}$ Department of Applied Mathematics and Modeling, Faculty of Mathematics and Informatics, \\ Paisii Hilendarski University of Plovdiv, 24 Tsar Assen Street, 4000 Plovdiv, Bulgaria \\ ${ }^{3}$ Metal Vapour Lasers Department, Georgi Nadjakov Institute of Solid State Physics, \\ Bulgarian Academy of Sciences, 72 Tsarigradsko Shaussee Boulevard, 1784 Sofia, Bulgaria
}

Correspondence should be addressed to Snezhana Georgieva Gocheva-Ilieva, snegocheva@yahoo.com

Received 17 April 2009; Accepted 3 August 2009

Recommended by Saad A Ragab

\begin{abstract}
An improved theoretical model of the gas temperature profile in the cross-section of an ultraviolet copper ion excited copper bromide laser is developed. The model is based on the solution of the one-dimensional heat conduction equation subject to special nonlinear boundary conditions, describing the heat interaction between the laser tube and its surroundings. It takes into account the nonuniform distribution of the volume power density along with the radius of the laser tube. The problem is reduced to the boundary value problem of the first kind. An explicit solution of this model is obtained. The model is applied for the evaluation of the gas temperature profiles of the laser in the conditions of free and forced air-cooling. Comparison with other simple models assumed constant volume power density is made. In particular, a simple expression for calculating the average gas temperature is found.
\end{abstract}

Copyright (C) 2009 Iliycho Petkov Iliev et al. This is an open access article distributed under the Creative Commons Attribution License, which permits unrestricted use, distribution, and reproduction in any medium, provided the original work is properly cited.

\section{Introduction}

Deep ultraviolet (DUV) gas laser sources have been objects of great interest in the recent years, because of a wide variety of applications, such as high-precision processing of different materials, high-resolution laser lithography in microelectronics, high-density optical recording of information, laser-induced modification in various materials newly developed, as well as laser-induced fluorescence in plasma and wide-gap semiconductors [1-7]. These applications require a DUV laser source in order to achieve the necessarily high resolution 
or to induce some phenomena in different materials, such as material and its surface modification, image recording, and fluorescence. The photon energy is sufficient first, to produce photochemical ablation rather than melting and subsequent vaporization, or second, to initiate the induced processes. The DUV laser ablation produces clean precision cutting and drilling with a minimum of thermal or mechanical damages on the target. DUV beams, in addition to heatless etching, offer the advantage of focusing with less limitation due to diffraction. The good spatial structure of the laser beam is generally important for the goodquality image projected, while narrow linewidth would reduce the chromatic aberrations. Chromatic aberrations are very difficult to correct because of the limited number of optical materials that transmit in the DUV range. The excimer lasers, which are generally used as light sources in the DUV range, can neither reach the spatial structure nor the linewidth required. Their wide application is also impeded by their cost and considerable overheads. Lately, there has been widespread interest in the ion metal vapor lasers, operating below $300 \mathrm{~nm}$ in the DUV spectral region. High beam quality and narrow-linewidth are inherent for metal vapor lasers. For the first time laser oscillation on four DUV Cu${ }^{+}$lines: $248.6 \mathrm{~nm}$, $252.9 \mathrm{~nm}, 260.0 \mathrm{~nm}$, and $270.3 \mathrm{~nm}$, in a nanosecond, pulsed longitudinal Ne-CuBr discharge was obtained in [1]. In [2] an active zone diameter scanning of the DUV $\mathrm{Cu}^{+} \mathrm{Ne}-\mathrm{CuBr}$ laser was carried out and the optimal discharge conditions were found for each active zone diameter. A record average output power of $1.3 \mathrm{~W}$ was obtained at multiline operation. A record specific average output power was $57 \mathrm{~mW} / \mathrm{cm}^{3}$ at an active volume of $23 \mathrm{~cm}^{3}$. The highest peak pulse power and average laser power on the $248.6 \mathrm{~nm}$ laser line for the DUV $\mathrm{Cu}^{+}$lasers were $3.25 \mathrm{~W}$ and $0.85 \mathrm{~W}$, respectively.

For the metal and metal halide vapor lasers, the thermal mode, and in particular the radial temperature distribution, is of great importance for the stability of the laser operation and for the achievement of high output characteristics as well $[8,9]$. In addition, crosssections and rate constants for heavy particle collisions, which thoroughly determine the inverse population and laser output parameters, such as asymmetric charge transfer, Penning ionization, depend strongly on the gas temperature. That is why the radial temperature distribution in the active zone of the DUV $\mathrm{Cu}^{+} \mathrm{Ne}-\mathrm{CuBr}$ laser is object of a detailed investigation. The gas temperature problem is also topical in radio-frequency discharges, widely encountered in gas lasers, electron and ion technologies, nanotechnology, plasmachemical technology, and more [8].

It is well known that the experimental techniques for gas temperature measurement, using spectral lines broadening and thermocouple are definitely imprecise. That is why mathematical and computer models are applied to carry out the temperature distribution. These models are used not only to evaluate the temperature but also for complex modeling of plasma kinetics processes [9-14].

The determination of the gas temperature profile in metal vapor lasers has been firstly considered in [9], where self-consistent mathematical models have been developed based on an exact solution of the one-dimensional steady-state heat conduction equation subject to the simple boundary conditions of the first and second kind. Till now, the same method has been used in all other papers in this area, when the buffer gas temperature is calculated, see for instance, [9-16]. In these studies, the expected average temperature and profile of the gas are obtained using the following simplifying assumptions: (1) the average power input is considered uniform in the discharge-no radial or longitudinal variations in power density are considered ( $q_{v}=$ constant $)$; $(2)$ the wall temperature is assumed to be constant.

However, in practice, and in computer modeling and simulations, these assumptions are not fully applicable. That is so because the real values of the volume power density $q_{v}$ 


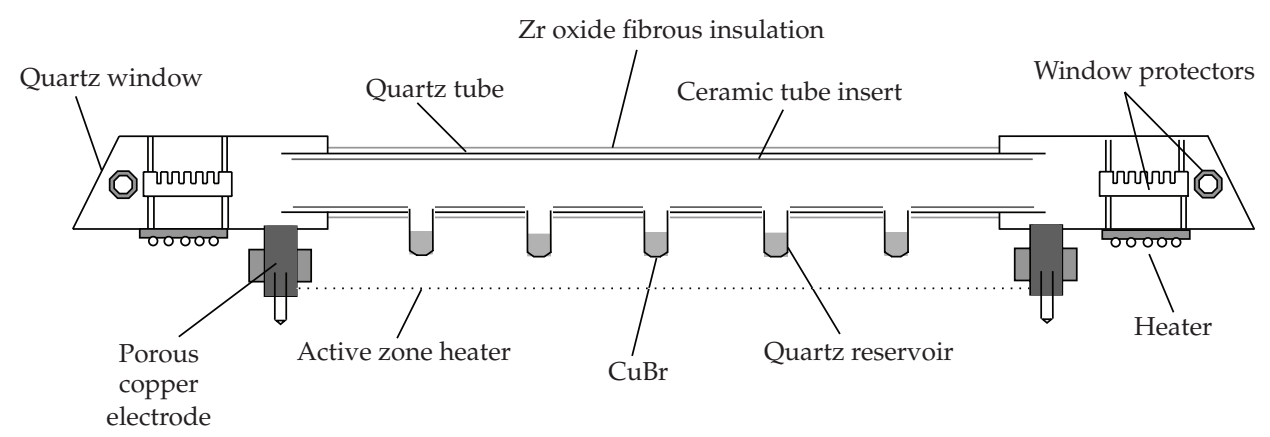

Figure 1: Construction of discharge tube.

are not uniformly distributed and vary strongly along the tube radius taking its maximum in the central axis of the discharge. In addition, the temperature of the outer surface of the laser tube under insulation is unknown and will change with variation of the laser geometry, input electric power, and other laser parameters including temperature of the surroundings.

In $[17,18]$, the temperature profile in the case of a copper bromide vapor laser with wavelength $510.6 \mathrm{~nm}$ and $578.2 \mathrm{~nm}$ was determined by using a new approach. It is based on solving the heat conduction equation with $q_{v}=$ constant at nonlinear boundary value conditions for a given temperature of the surroundings.

In this study, for the first time, the analytical investigation of the temperature profile in the cross-section of the laser tube is performed with the assumption of a specified qualitative distribution of $q_{v}$, dependent on the tube radius, namely, $q_{v}=q_{v}(r)$. Using the approach similar to this in $[17,18]$, in the case of $\mathrm{UV} \mathrm{Cu} \mathrm{Cu}^{+} \mathrm{CuBr}$ lasers, a new improved analytical model consisting of the one-dimensional heat conduction equation, subject to nonlinear boundary conditions of the third and fourth kind is derived. At a given air temperature of the surroundings, due to the heat convection and heat radiation, the proposed model allows to take into account the heat exchange processes between the outer surface of the laser tube and its surroundings. The gas temperature profile in the tube and the wall temperature are expressed by an explicit solution of the obtained problem and are directly dependent on the basic input laser parameters. The model is applied for evaluating the natural and forced aircooling of the laser tube.

\section{Experimental Setup}

The construction of the gas-discharge laser tube described by the model is presented in Figure 1 . The basic tube with an $18 \mathrm{~mm}$ inside diameter and $24.5 \mathrm{~mm}$ outside diameter is made of fused quartz. The active length is $86 \mathrm{~cm}$. The $\mathrm{CuBr}$ powder is placed in five quartz side-arm reservoirs. A ceramic tube insert with an inside diameter of $5.2 \mathrm{~mm}$ is sleeved in the basic tube. In the ceramic insert, five holes are made over each reservoir for the $\mathrm{CuBr}$ vapor diffusion into the active zone. The optic cavity of the laser studied is formed by two dielectric-coated mirrors. Mirror separation is $1.8 \mathrm{~m}$.

The laser is excited by a pulsed electrical scheme with Interacting Circuits (IC). The IC excitation of $\mathrm{CuBr}$ lasers, operating on self-terminating copper atom transitions, was described in detail in [19]. 


\section{Description of the Mathematical Model}

The aforementioned multiline copper-bromide laser operates in the UV-region [1-5]. The total input electric power is $1300 \mathrm{~W}$. Taking into account the losses in power supply, the laser tube is fed with power $Q_{1}=1000 \mathrm{~W}$. The output multiline laser power is $500 \mathrm{~mW}$. The geometric design of the cross-section of the laser tube in the active zone is shown in Figure 2. The laser source is manufactured from quartz (3.2), in which a ceramic tube of $\mathrm{Al}_{2} \mathrm{O}_{3}$ (3.1) is inserted along with the active laser volume, and the quartz tube is covered from the outside active volume with extra heat-insulating wadding (3.3) made of felt-glass, mineral material, or zircon oxide.

The model is developed with the following assumptions: (i) the temperature profile is determined in a quasi-stationary regime; (ii) the gas temperature does not change substantially in the interimpulse period; (iii) the total input electric power $Q_{1}=1000 \mathrm{~W}$ in the active volume is transformed into heat, the power transferred to the walls as a result of the discharge radiation and the deactivation of the excited and charged particles is not taken into account; (iv) the thermal radiation of the heated gas in the active volume is ignored.

The temperature distribution $T_{g}$ in the cross-section of the laser tube is governed by the following quasi steady-state two-dimensional heat conduction equation:

$$
\operatorname{div}\left(\lambda_{g} \operatorname{grad} T_{g}\right)+q_{v}=0
$$

where $\lambda_{g}$ is the thermal conductivity of the gas (here neon) and $q_{v}$ is the volume density of the discharge. Due to the radial symmetry, $T_{g}$ depends only on the variable $r$ along with the radius of the tube. Consequently, in cylindrical configuration, (3.1) is reduced to the form

$$
\frac{1}{r} \frac{d}{d r}\left(r \lambda_{g} \frac{d T_{g}(r)}{d r}\right)+q_{v}=0, \quad 0 \leq r<R_{1}, R_{1}=\frac{d_{1}}{2}
$$

Usually, as it was mentioned earlier, equation (3.1) (resp., (3.2)) is solved in publications under the boundary conditions:

$$
T_{g}\left(R_{1}\right)=T_{w},\left.\quad \frac{d T_{g}(r)}{d r}\right|_{r=0}=0,
$$

where $T_{w}$ is the measured temperature of the outer wall of the tube (under insulation) [9-13]. Commonly, $\lambda_{g}$ is in the form $\lambda_{g}=\lambda_{0} T_{g}^{m}$.

In the case $q_{v}=$ constant, (3.2) possesses an exact solution [9], written in the form

$$
T_{g}(r)=\left[T_{w}^{m+1}+\frac{q_{v}(m+1)}{4 \lambda_{0}}\left(R^{2}-r^{2}\right)\right]^{1 /(m+1)} .
$$




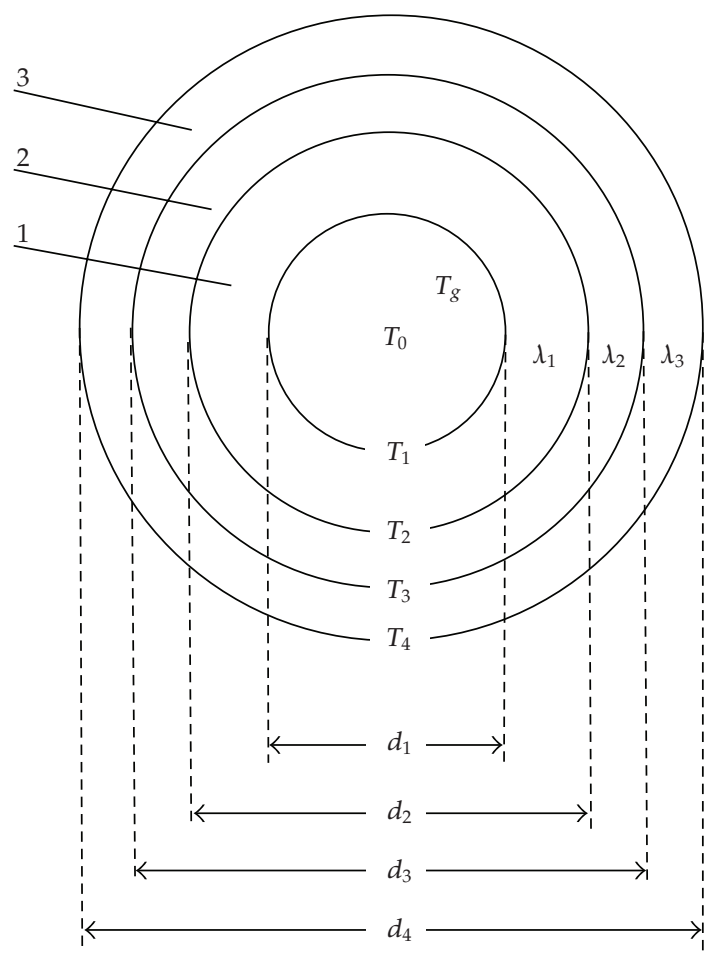

Figure 2: Principle geometrical design of the cross-section of the laser source: (1) ceramic $\left(\mathrm{Al}_{2} \mathrm{O}_{3}\right)$ tube; (2) quartz tube; (3) thermal insulation. The diameters are, respectively: $d_{1}=5.2 \mathrm{~mm}, d_{2}=18 \mathrm{~mm}, d_{3}=$ $24.5 \mathrm{~mm}$, and $d_{4}=32.5 \mathrm{~mm}$.

For solving (3.2), we need to obtain the correct boundary conditions corresponding to (3.3) for $r=R_{1}$ (see Figure 2):

$$
T_{g}\left(R_{1}\right)=T_{1},\left.\quad \frac{d T_{g}(r)}{d r}\right|_{r=0}=0 .
$$

For that purpose, we will apply the distribution of the radial heat flow through the composite laser tube. We consider the following mixed boundary conditions of the third and fourth kind in cylindrical configuration [20-22]:

$$
\begin{gathered}
T_{1}=T_{2}+\frac{q_{l} \ln \left(d_{2} / d_{1}\right)}{2 \pi \lambda_{1}}, \quad T_{2}=T_{3}+\frac{q_{l} \ln \left(d_{3} / d_{2}\right)}{2 \pi \lambda_{2}}, \quad T_{3}=T_{4}+\frac{q_{l} \ln \left(d_{4} / d_{3}\right)}{2 \pi \lambda_{3}}, \\
Q_{1}=\alpha F_{4}\left(T_{4}-T_{\text {air }}\right)+F_{4} \varepsilon c\left[\left(\frac{T_{4}}{100}\right)^{4}-\left(\frac{T_{\text {air }}}{100}\right)^{4}\right]
\end{gathered}
$$

where $T_{j}$ denotes the temperatures at the boundaries of the tubes, $j=1, \ldots, 4$, respectively (see Figure 2).

Boundary conditions (3.6) express the equation of the continuity of the heat flow at the borders of the three mediums of the composed tube. Here $q_{l}$ is the power per unit length, 
Table 1: Related parameters of the theoretical calculation.

\begin{tabular}{|c|c|c|c|c|}
\hline Parameter & Value & Description & Condition & Reference \\
\hline$Q_{1}$ & $1000 \mathrm{~W}$ & $\begin{array}{l}\text { Effective input electric } \\
\text { power }\end{array}$ & & {$[5,6]$} \\
\hline$l_{a}$ & $0.86 \mathrm{~m}$ & Length of the active zone & & {$[5,6]$} \\
\hline$q_{0}$ & $54.6 \mathrm{Wcm}^{-3}$ & constant & & {$[5,6]$} \\
\hline$q_{l}$ & $1162.8 \mathrm{~W} / \mathrm{m}$ & $\begin{array}{l}\text { Electric power per unit } \\
\text { length }\end{array}$ & & {$[5,6]$} \\
\hline$\lambda_{0}$ & 0.0010029 & $\begin{array}{l}\text { Specific coefficient in } \lambda_{g}, \\
\text { Equations (3.1), (3.2) }\end{array}$ & & [20] \\
\hline$m$ & 0.6817 & $\begin{array}{l}\text { Power coefficient for } \\
\text { neon in } \lambda_{g} \text {, Equations } \\
(3.1),(3.2)\end{array}$ & & \\
\hline$\lambda_{1}$ & $2.08 \mathrm{~W} / \mathrm{mK}$ & $\begin{array}{l}\text { Thermal conductivity of } \\
\text { the ceramic tube }\end{array}$ & $T=800 / 1100 \mathrm{~K}$ & [22] \\
\hline$\lambda_{2}$ & $1.96 \mathrm{~W} / \mathrm{mK}$ & $\begin{array}{l}\text { Thermal conductivity of } \\
\text { the quartz tube }\end{array}$ & $T=800 / 1100 \mathrm{~K}$ & {$[22]$} \\
\hline$\lambda_{3}$ & $1.78 \mathrm{~W} / \mathrm{mK}$ & $\begin{array}{l}\text { Thermal conductivity of } \\
\text { the thermal insulation }\end{array}$ & $T=800 / 1100 \mathrm{~K}$ & {$[22]$} \\
\hline$\varepsilon$ & 0.72 & $\begin{array}{l}\text { Integral emissivity of the } \\
\mathrm{Zr} \text { oxide insulation }\end{array}$ & & {$[20,22]$} \\
\hline c & $5.67 \mathrm{Wm}^{-2} \mathrm{~K}^{-4}$ & $\begin{array}{l}\text { Black body radiation } \\
\text { coefficient }\end{array}$ & & [23] \\
\hline$T_{\text {air }}$ & $300 \mathrm{~K}$ & Temperature of the air & & \\
\hline$\lambda_{\text {air }}$ & $0.0251 \mathrm{~W} / \mathrm{mK}$ & $\begin{array}{l}\text { Thermal conductivity of } \\
\text { the air }\end{array}$ & $T_{\text {air }}=300 \mathrm{~K}$ & {$[22,23]$} \\
\hline$v_{\text {air }}$ & $15.7 \times 10^{-6} \mathrm{~m}^{2} / \mathrm{s}$ & $\begin{array}{l}\text { Kinematical viscosity of } \\
\text { the air }\end{array}$ & $T_{\text {air }}=300 \mathrm{~K}$ & {$[22,23]$} \\
\hline$\beta_{\text {air }}$ & $3.14 \times 10^{-3} \mathrm{~K}^{-1}$ & $\begin{array}{l}\text { Coefficient of cubical heat } \\
\text { expansion of the air }\end{array}$ & $T_{\text {air }}=300 \mathrm{~K}$ & {$[22,23]$} \\
\hline$g$ & $9.80665 \mathrm{~ms}^{-2}$ & Gravitational acceleration & & [23] \\
\hline$v$ & $20 \mathrm{~m} / \mathrm{s}$ & $\begin{array}{l}\text { Velocity of the moving } \\
\text { fluid }\end{array}$ & & \\
\hline
\end{tabular}

$q_{l}=Q_{1} / l_{a} ; l_{a}$ is the active length $[2,5,6] ; \lambda_{j}, j=1,2,3$ are the thermal conductivities of the $\mathrm{Al}_{2} \mathrm{O}_{3}$ tube, quartz tube and the thermal insulation, respectively; $d_{j}, j=1, \ldots, 4$ are the diameters of the composite tubes (see Figure 2 and Table 1).

The boundary condition (3.7) shows the heat exchange between the outer surface of the laser tube and the surroundings. The first term on the right-hand side of (3.7) evolves from Newton-Riemann's law for heat exchange by convection. The second term represents the Stefan-Boltzmann law for heat exchange by radiation. The value of $Q_{1}$ is equal to the electric power of $1000 \mathrm{~W}$, in accordance with assumption (iii), as it was stated earlier, $\alpha$ is the heat transfer coefficient, $F_{4}$ is the outside area of the insulation, $\varepsilon$ is the integral emissivity of the material, $c$ is the black body radiation coefficient, and $T_{\text {air }}$ is the temperature of the air.

The two unknown values $\alpha$ and $T_{4}$ in boundary condition (3.7) have to be determined. The values of the constants used in this study are given in Table 1.

In this way we obtain the temperature model described by (3.2) and boundary conditions (3.6)-(3.7), equivalent to (3.2), (3.5). Our aim is to find an analytical formula for the solution of this model at $q_{v}=q_{v}(r)$. 


\section{Determination of the Gas Temperature $T_{g}(r)$ at Radial Distribution of the Volume Power Density $q_{v}(r)$}

In this section, we will obtain an explicit solution for the gas temperature $T_{g}(r)$ satisfying the proposed theoretical model (3.2), (3.6), (3.7) and will discuss its application.

\subsection{Determining the Variable Radial Distribution $q_{v}=q_{v}(r)$}

Due to the lack of experimental data for $q_{v}=q_{v}(r)$, we will derive it as a qualitative theoretical dependency. From $q_{v}=j E$ and $j \approx \sigma E$, we have $q_{v} \approx \sigma E^{2}$, where $E=E(r)$ is the electric field intensity and $\sigma$ is the electrical conductivity of the medium. In [24] the distribution of the field intensity in the cross-section of the tube is represented by the expression $E(r)=$ $E_{0} J_{0}\left(\left(2.4 / R_{1}\right) r\right)$, where $J_{0}\left(\left(2.4 / R_{1}\right) r\right)$ is a Bessel function of the first kind, $0 \leq r \leq R_{1}$. In this way, we have $q_{v}(r)=Q_{0}\left[J_{0}\left(\left(2.4 / R_{1}\right) r\right)\right]^{2}$, where $Q_{0}$ is a constant, which is found below. The Bessel function $J_{0}$ is well known and usually represented in [23, a table] in what follows. In this form, it is not suitable for direct engineering-physics calculations. For this reason, we will approximate the term $\left[J_{0}\left(\left(2.4 / R_{1}\right) r\right)\right]^{2}$ by a polynomial of the third-degree $\left[J_{0}(x)\right]^{2} \approx$ $a_{1}+a_{2} x+a_{3} x^{2}+a_{4} x^{3}$, where $x=\left(2.4 / R_{1}\right) r$. Based on tabular data from [23] and using the least squares method, we find

$$
a_{1}=1.0044, \quad a_{2}=-0.01768, \quad a_{3}=-0.5657, \quad a_{4}=0.1668 .
$$

For $q_{v}(r)$, we obtain:

$$
\begin{aligned}
q_{v}(r) & =Q_{0}\left[a_{1}+a_{2} \frac{2.4}{R_{1}} r+a_{3}\left(\frac{2.4}{R_{1}}\right)^{2} r^{2}+a_{4}\left(\frac{2.4}{R_{1}}\right)^{3} r^{3}\right] \\
& =Q_{0}\left(a_{1}+B r+C r^{2}+D r^{3}\right),
\end{aligned}
$$

where

$$
B=a_{2} \frac{2.4}{R_{1}}, \quad C=a_{3}\left(\frac{2.4}{R_{1}}\right)^{2}, \quad D=a_{4}\left(\frac{2.4}{R_{1}}\right)^{3}
$$

The constant $Q_{0}$ can be found by using the equality of areas, bounded between the graphics of each of the functions $q_{0}=$ constant and $q_{v}=q_{v}(r)$, and the abscissa $r$ (see Figure 3):

$$
2 q_{0} R_{1}=2 Q_{0} \int_{0}^{R_{1}}\left(a_{1}+B r+C r^{2}+D r^{3}\right) d r .
$$

After integrating in (4.4) and substituting the values of the constants, we find

$$
Q_{0} \approx 2.131 q_{0} .
$$

In Figure 3, the distribution of the volume power densities $q_{0}=$ constant and $q_{v}=$ $q_{v}(r)$, according to (4.2) and (4.5), are illustrated in relative units, assuming here, in order to 
simplify that $q_{0}=$ constant $=1$. From Figure 3 and (4.5), it can be observed that in the center of the discharge the local electrical volume power density for (4.2) is over two times larger than $q_{v}=$ constant. This suggests a difference in the distribution of $T_{g}(r)$ in the two cases being examined: $q_{v}=$ constant and $q_{v}=q_{v}(r)$.

\subsection{Determining the Gas Temperature $T_{g}(r)$}

The solution to (3.2) at mixed boundary conditions (3.6)-(3.7) and radial distribution $q_{v}(r)$ of type (4.2) has the following form:

$$
T_{g}(r)=\left\{T_{1}^{m+1}+\frac{(m+1) Q_{0}}{\lambda_{0}}\left[\frac{a_{1}}{4}\left(R_{1}^{2}-r^{2}\right)+\frac{B}{9}\left(R_{1}^{3}-r^{3}\right)+\frac{C}{16}\left(R_{1}^{4}-r^{4}\right)+\frac{D}{25}\left(R_{1}^{5}-r^{5}\right)\right]\right\}^{1 /(m+1)}
$$

where the constants $B, C, D$ were introduced in (4.3).

Detailed determination of (4.6) is given in the appendix.

\subsection{Application of the Mathematical Model}

The obtained explicit solution (4.6) can be used when the value $T_{1}$ of the temperature of the internal tube is known. There are two cases as follows.

(1) The temperature $T_{3}$ of the outside surface of the laser tube (i.e., quartz tube) under the insulation is known (see Figure 2). For existing laser devices, it can be measured, for instance, by a thermocouple. Then $T_{2}$ and $T_{1}$ can be calculated by means of the corresponding boundary conditions from (3.6).

(2) The temperature $T_{3}$ is unknown. This problem can arise in the development of new laser sources or implementation of different computer simulations. In this case, the temperature of the surroundings $T_{\text {air }}$ must be specified, usually $T_{\text {air }}=300 \mathrm{~K}$. To use boundary condition (3.7) we need to find $\alpha$ and $T_{4}$. In the following section, we discuss the procedure for determining the heat transfer coefficient $\alpha$ and obtaining a nonlinear algebraic equation for the temperature $T_{4}$. Then, applying (3.6), we calculate $T_{3}, T_{2}$, and $T_{1}$.

\section{Evaluation of Cooling and Discussion}

We will apply the derived temperature model (3.2), (3.6)-(3.7) for determining the gas temperature in the cases of free and forced convection.

\subsection{Cooling of the Laser Tube by Free Convection}

In [18], a simplified temperature model in the case of free convection at $q_{v}=$ constant has been used. Here we will compare the results obtained by our new model for the general case $q_{v}=q_{v}(r)$ with those in [18]. 


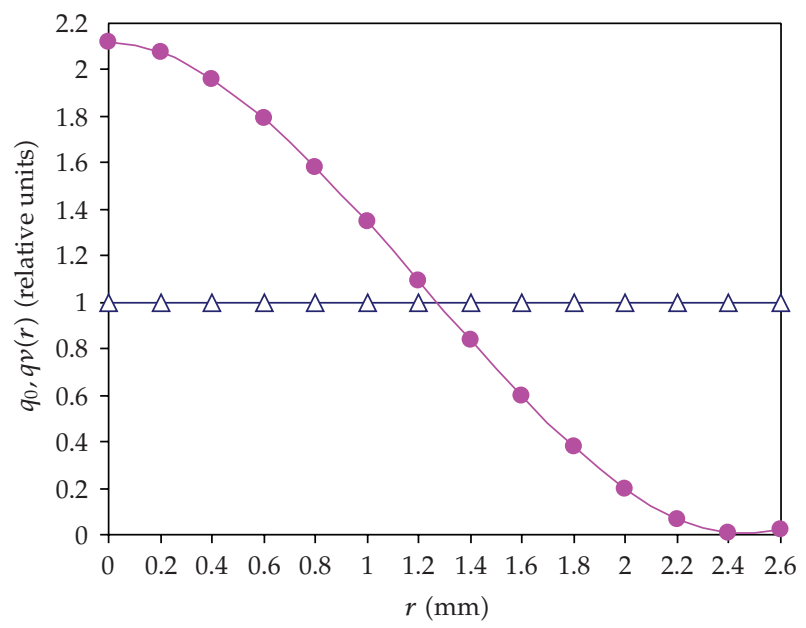

Figure 3: Distribution of volume power density along the half cross-section of the internal tube, in relative units: $\triangle: q_{v}=q_{0}=1, \bullet: q_{v}=q_{v}(r)$.

In the case of free convection, the heat transfer coefficient $\alpha$ in (3.7) was calculated in [18] as

$$
\alpha=0.46 \lambda_{\text {air }}\left[\frac{g \beta_{\text {air }} d_{4}^{3}\left(T_{4}-T_{\text {air }}\right)}{v_{\text {air }}^{2}}\right]^{0.25} / d_{4}
$$

The substitution of (5.1) in boundary condition (3.7) with consequent representation in relation to the power per unit length $q_{l}$ results in [18]

$$
q_{l}=0.46 \pi \lambda_{\text {air }}\left[\frac{g \beta_{\text {air }} d_{4}^{3}\left(T_{4}-T_{\text {air }}\right)}{v_{\text {air }}^{2}}\right]^{0.25}\left(T_{4}-T_{\text {air }}\right)+\pi d_{4} \varepsilon c\left[\left(\frac{T_{4}}{100}\right)^{4}-\left(\frac{T_{\text {air }}}{100}\right)^{4}\right] .
$$

In the previous expressions (5.1)-(5.2), the numerical values of $g, \beta_{\text {air }}, v_{\text {air }}$, and $\lambda_{\text {air }}$ are given in Table 1 . The data is correct for air temperature $T_{\text {air }}=300 \mathrm{~K}$ [22].

However (5.2) is a nonlinear equation with respect to the outside temperature of the laser device insulation- $T_{4}$. Also (5.2) can be easily solved by any computer algebra system, for instance by Mathematica [25]. Once the temperature $T_{4}$ is calculated, the values of $T_{3}$, $T_{2}$, and $T_{1}$ can be evaluated from (3.6), and the gas temperature $T_{g}(r)$ in the internal tube is determined by (4.6).

In Figure 4, on the same coordinate system, the distributions of the gas temperature $T_{g}(r)$ in the cross-section along with the radius of the laser tube for $q_{v}=$ constant and $q_{v}=q_{v}(r)$ are presented. In Table 2, special characteristic temperatures $T_{4}, T_{3}, T_{2}, T_{1}$, and the maximum temperature $T_{0}$ in the center of the laser tube are given (see also Figure 1).

Table 2 shows that temperatures $T_{4}, T_{3}, T_{2}$, and $T_{1}$ are equal. Their values are determined by the total electrical power emitted within the active volume and are independent from its radial distribution. In both cases, this power is the same- $1000 \mathrm{~W}$. Table 2 and Figure 4 show that $T_{0}=T_{g}(0)=T_{\max }$ when $q_{v}=q_{v}(r)$ is $90^{\circ} \mathrm{C}$ higher than the 
Table 2: Gas temperature at special characteristic points in the case of free convection.

\begin{tabular}{lccccc}
\hline & $T_{4}(\mathrm{~K})$ & $T_{3}(\mathrm{~K})$ & $T_{2}(\mathrm{~K})$ & $T_{1}(\mathrm{~K})$ & $T_{0}(\mathrm{~K})$ \\
\hline$q_{v}=q_{0}=$ constant & 672.6 & 688.3 & 717.4 & 838.3 & 1573.9 \\
$q_{v}=q_{v}(r)$ & 672.6 & 688.3 & 717.4 & 838.3 & 1663.9 \\
\hline
\end{tabular}

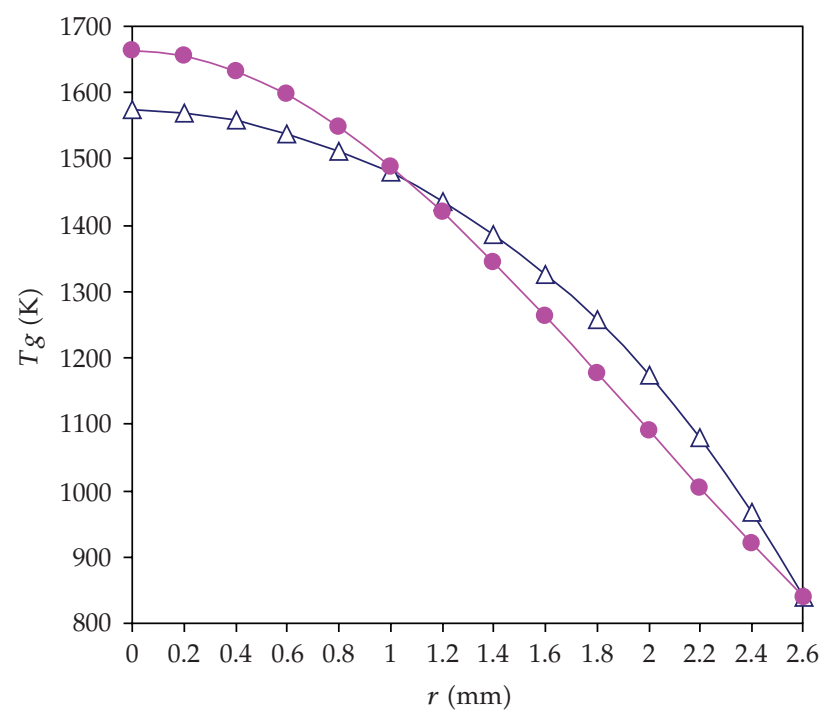

Figure 4: Gas temperature distribution along the half cross-section of the internal tube with free convection: $\triangle: q_{v}=q_{0}=54.6 \mathrm{~W} \mathrm{~cm}^{-3}, \bullet: q_{v}=q_{v}(r)$ from $(4.2)$.

corresponding value when $q_{v}=$ constant. The results for the gas temperature in the case of $q_{v}=$ constant have the same behavior as the calculated values in $[9,11]$.

\subsection{Cooling of the Laser Tube by Forced Convection}

For all types of convection, the Nusselt criterion, $\mathrm{Nu}=\alpha H / \lambda$, holds [20-22], from which for $H=d_{4}$ and $\lambda=\lambda_{\text {air }}$, we find

$$
\alpha=\frac{\mathrm{Nu \lambda _{ \text {air } }}}{d_{4}} .
$$

In the case of forced convection, the Reynolds criterion is represented by [20, 21]

$$
\operatorname{Re}=\frac{v \cdot l_{a}}{v_{\text {air }}}
$$

where $v$ is the velocity of the moving fluid, $l_{a}$ is the length of the laser tube and $v_{\text {air }}$ is the kinematical viscosity of the air. However (5.4) is valid for $40<\operatorname{Re}<4000$ [22].

For horizontal tubes with forced air cooling the following equality holds [22]:

$$
\mathrm{Nu}=0.615 \mathrm{Re}^{0.466} .
$$


Table 3: Gas temperature at special characteristic points in the case of forced convection.

\begin{tabular}{lccccc}
\hline & $T_{4}(\mathrm{~K})$ & $T_{3}(\mathrm{~K})$ & $T_{2}(\mathrm{~K})$ & $T_{1}(\mathrm{~K})$ & $T_{0}(\mathrm{~K})$ \\
\hline$q_{v}=q_{0}=$ constant & 459.3 & 475.0 & 504.1 & 625.0 & 1443.6 \\
$q_{v}=q_{v}(r)$ & 459.3 & 475.0 & 504.1 & 625.0 & 1539.0 \\
\hline
\end{tabular}

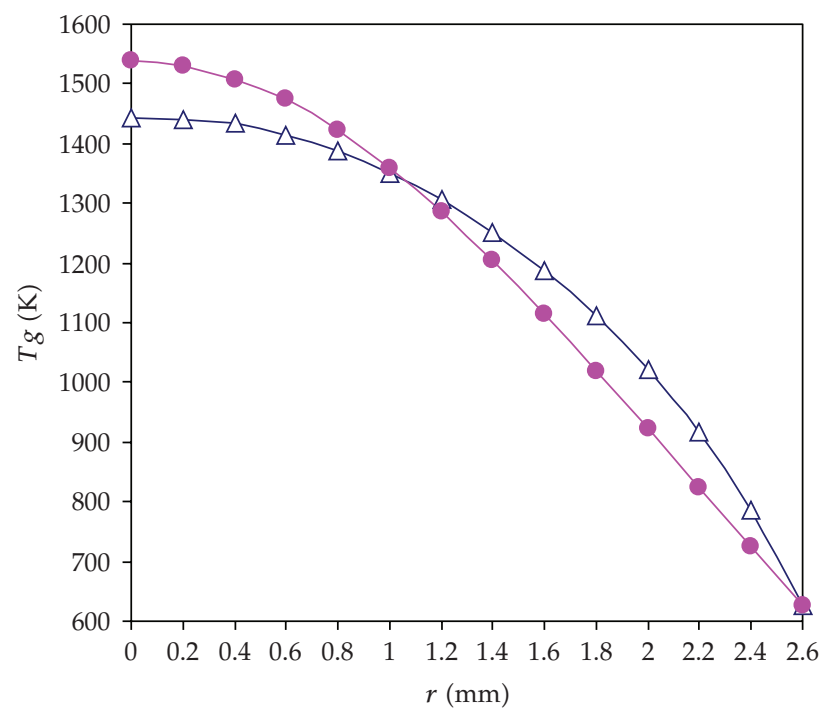

Figure 5: Gas temperature distribution along the half cross-section of the internal tube in the case of forced convection, $v=20 \mathrm{~m} / \mathrm{s}: \Delta: q_{v}=q_{0}=54.6 \mathrm{~W} \mathrm{~cm}^{-3}, \bullet: q_{v}=q_{v}(r)$ from (4.2).

From (5.3), (5.4), and (5.5), the heat transfer coefficient $\alpha$ is

$$
\alpha=0.615 \frac{\lambda_{\text {air }}}{d_{4}}\left(\frac{v d_{4}}{v_{\text {air }}}\right)^{0.466}
$$

Substituting (5.6) in boundary condition (3.7) and representing it with respect to the power per unit length $q_{l}$, we obtain a nonlinear algebraic equation for $T_{4}$ in the form

$$
q_{l}=0.615 \pi \lambda_{\text {air }}\left(\frac{v d_{4}}{v_{\text {air }}}\right)^{0.466} \cdot\left(T_{4}-T_{\text {air }}\right)+\pi d_{4} \varepsilon c\left[\left(\frac{T_{4}}{100}\right)^{4}-\left(\frac{T_{\text {air }}}{100}\right)^{4}\right]
$$

In this way by solving (5.7), we determine $T_{4}$. Then using (3.6) and (4.6), we find the required gas temperature profile in the cross-section of the laser tube.

The obtained values of some characteristic temperatures are given in Table 3, including the maximum value $T_{0}$ along the center of the tube. The results of the calculated values of $T_{g}(r)$ in the two cases $q_{v}=q_{0}=$ constant and $q_{v}=q_{v}(r)$ are shown in Figure 5 for air flow $v=20 \mathrm{~m} / \mathrm{s}$.

As it is expected the cooling process causes a decrease of the buffer gas temperature in relation with the case of free convection (compare Tables 2 and 3, and Figures 4 and 5). 
It can be noted that although the maximum local electric power at the center of the tube is twice higher for $q_{v}=q_{v}(r)$ (see Figure 3), the difference between the corresponding maximum temperatures is only $95^{\circ} \mathrm{C}$. This result is almost the same as in the case of free convection. The deviation is on average around $6 \%$. We can conclude that in principle, solution (3.4) can successfully be used to analyze temperature conditions of existing laser sources, when the temperature $T_{1}$ is known.

As an absolute quantity, the difference of $90 / 95^{\circ} \mathrm{C}$ at the center of the discharge should not be overlooked. The model discussed in this paper can better explain and predict the occurrence of a number of negative phenomena connected with the overheating of the laser medium. The increase of $90 / 95^{\circ} \mathrm{C}$ in the temperature at the center of the discharge can lead to a contraction of the gas discharge, thermal ionizing instability, and thermochemical gas degradation, additional thermal population of lower laser levels. In the end, this leads to decreased laser power and deterioration in mode composition. In some cases, the overheating of the discharge at the center of the tube may lead to a cessation of laser generation along its axis and the appearance of dark spots at the center of laser beam. For this reason, regardless the complexity of the new model, its use is fully advisable.

\section{Average Temperature in the Active Volume}

During the analysis of the temperature condition of existing or new laser devices, the average gas temperature in the active volume is a characteristic of great importance. It is defined as

$$
\bar{T}_{g}=\frac{1}{R_{1}} \int_{0}^{R_{1}} T_{g}(r) d r
$$

where $T_{g}(r)$ is the radial distribution of temperature in the active volume.

The average value of temperature $\bar{T}_{g}$ for equal configurations depends only on the electric power, supplied to the active volume, and is independent of the radial distribution of $T_{g}(r)$. In our case, the electrical power is $Q_{1}=1000 \mathrm{~W}$. The average temperature should not change for the temperature distribution of the type in (3.4) and (4.6).

All subsequent calculations have been made using the Mathematica software system

For the radial distribution of $T_{g}(r)$ given by formula (3.4), the result is

$$
\bar{T}_{g, \mathrm{Eq} \cdot(3.4)}=T_{1}\left(1+\frac{(m+1) q_{v} R_{1}^{2}}{4 \lambda_{0} T_{1}^{m+1}}\right)^{1 /(m+1)} \times\left[{ }_{2} F_{1}\left(\frac{1}{2},-\frac{1}{m+1} ; \frac{3}{2} ;\left(1+\frac{4 T_{1}^{m+1} \lambda_{0}}{(m+1) q_{v} T_{1}^{m+1}}\right)^{-1}\right)\right] .
$$

The numerical value of (6.2) is $\bar{T}_{g, \text { Eq. (3.4) }}=1347 \mathrm{~K}$.

For the radial distribution of $T_{g}(r)$ from (4.6) the quantity (6.1) does not have an exact algebraic solution and the integral in (6.1) is solved numerically. The numerical value is $\bar{T}_{g, \text { Eq.(4.5) }}=1339 \mathrm{~K}$. The approximate calculation leads to an insignificant deviation of values

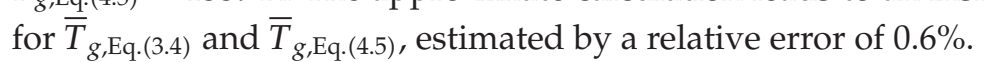

The presence of a hypergeometric function of type ${ }_{2} F_{1}(a, b ; c ; z)$ in the solution of (6.2) makes it practically difficult to use. For this reason, we represent the function ${ }_{2} F_{1}(a, b ; c ; z)$ 
by its Gauss series $[25,26]$, limited only to the first two terms of the expansion

$$
{ }_{2} F_{1}(a, b ; c ; z) \approx 1+\frac{a b z}{c} .
$$

The result is

$$
{ }_{2} F_{1}\left(\frac{1}{2},-\frac{1}{m+1} ; \frac{3}{2} ;\left(1+\frac{4 T_{1}^{m+1} \lambda_{0}}{(m+1) q_{v} T_{1}^{m+1}}\right)^{-1}\right) \approx 1-\frac{q_{v} R_{1}^{2}}{3\left[(m+1) q_{v} R_{1}^{2}+4 \lambda_{0} T_{1}^{m+1}\right]}
$$

In this way by using (6.2), we obtain

$$
\bar{T}_{g} \approx T_{1}\left(1+\frac{(m+1) q_{v} R_{1}^{2}}{4 \lambda_{0} T_{1}^{m+1}}\right)^{1 /(m+1)}\left(1-\frac{q_{v} R_{1}^{2}}{3\left[(m+1) q_{v} R_{1}^{2}+4 \lambda_{0} T_{1}^{m+1}\right]}\right) .
$$

The average value of the temperature from $(6.5)$ is $\bar{T}_{g}=1358 \mathrm{~K}$, with a relative error of $1 \%$. This shows that (6.5) can be used with sufficient accuracy to determine the average temperature in the active volume: $\bar{T}_{g} \approx \bar{T}_{g, \text { Eq.(3.4) }}$.

\section{Conclusion}

A theoretical mathematical model for evaluating the buffer gas temperature $T_{g}(r)$ in UV $\mathrm{Cu}^{+} \mathrm{Ne}-\mathrm{CuBr}$ laser is developed. It takes into account the nonuniformly distributed electrical power along the cross-section of the laser tube. Based on common theoretical dependencies, a suggestion is made for the qualitative distribution of volume power density $q_{v}=q_{v}(r)$. The model includes a heat conduction equation subject to nonlinear boundary conditions. An explicit solution with these conditions is obtained. The model is applied in the cases of free and forced convection. A simple expression is established for calculating the average gas temperature in the active volume.

An evaluation of the previous existing solution has been presented, describing the distribution of the gas temperature $T_{g}(r)$ under the assumption $q_{v}=$ constant. It has been established that, for such an assumption, the error when determining $T_{g}(r)$ at the center of the tube is about $6 \%$.

The obtained results at $q_{v}=$ constant have been compared with similar calculated results by using simple mathematical gas temperature models $[9,11]$.

A comparison has been made between the obtained temperature profiles of $T_{g}(r)$ at $q_{v}=$ constant and $q_{v}=q_{v}(r)$. It has been established that at the center of the tube the temperature when $q_{v}=q_{v}(r)$ is about $90-95^{\circ} \mathrm{C}$ higher, both for free and forced convection.

It has to be noted that the simple model when $q_{v}=$ constant cannot be used to evaluate radial buffer gas temperature when the temperature of the wall is unknown. The advantage of the model presented here is that when specific geometric dimensions have been chosen, the temperature of the surroundings and other parameters, the temperature $T_{3}$ of the outer wall of the laser tube is calculated, after which the values for the gas temperature within the tube are calculated. Therefore, the new model can be applied not only to precise the existing models but to design new laser devices as well. 
The proposed theoretical model and methodology could be useful for solving similar engineering problems where the calculation of the radial heat flow with an internal heat source is required. It gives the opportunity to carry out further computer simulations in order to optimize laser generation, while changing the geometrical design, tube materials, heat insulation, input electric power, and laser operating conditions.

\section{Appendix}

Let us consider the problem (3.2), (3.5), assuming that the temperature $T_{1}$ is determined. For $\lambda_{g}=\lambda_{0} T_{g}^{m}, 0 \leq r<R_{1}$ and $q_{v}=q_{v}(r),(3.2)$ becomes

$$
\frac{1}{r} \frac{d}{d r}\left(r \lambda_{0} T_{g}^{m} \frac{d T}{d r}\right)+q_{v}=0
$$

By the change of variable

$$
U(r)=T_{g}^{m+1}
$$

we have $T_{g}^{m}\left(d T_{g} / d r\right)=(1 / m+1)(d U / d r)$. After some simplification in (A.1) and (3.3), we obtain the problem

$$
\begin{aligned}
& \frac{d^{2} U}{d r^{2}}+\frac{1}{r} \frac{d U}{d r}+\frac{q_{v}(m+1)}{\lambda_{0}}=0, \\
& U\left(R_{1}\right)=T_{1}^{m+1},\left.\quad \frac{d U}{d r}\right|_{r=0}=0 .
\end{aligned}
$$

By introducing a new variable

$$
\tau=\frac{d U}{d r}
$$

However (A.3) can be rearranged in the form

$$
d(r \tau)+\frac{m+1}{\lambda_{0}} q_{v} r d r=0
$$

The integration of (A.6) and substitution of $q_{v}$ by the expression (4.2) yields $\tau r+((m+$ 1) $\left.Q_{0} / \lambda_{0}\right) \int\left(a_{1} r+B r^{2}+C r^{3}+D r^{4}\right) d r=C_{1}$, where $C_{1}$ is a constant of integration. We find

$$
\tau+\frac{(m+1) Q_{0}}{\lambda_{0}}\left(a_{1} \frac{r}{2}+B \frac{r^{2}}{3}+C \frac{r^{3}}{4}+D \frac{r^{4}}{5}\right)=\frac{C_{1}}{r} .
$$


Coming back to the variable $U$ according to (A.5), we obtain

$$
\frac{d U}{d r}+\frac{(m+1) Q_{0}}{\lambda_{0}}\left(a \frac{r}{2}+B \frac{r^{2}}{3}+C \frac{r^{3}}{4}+D \frac{r^{4}}{5}\right)=\frac{C_{1}}{r}
$$

The second boundary condition in (A.4) requires that $C_{1}=0$, so

$$
d U+\frac{(m+1) Q_{0}}{\lambda_{0}}\left(a_{1} \frac{r}{2}+B \frac{r^{2}}{3}+C \frac{r^{3}}{4}+D \frac{r^{4}}{5}\right) d r=0
$$

The integration of (A.9) yields

$$
U+\frac{(m+1) Q_{0}}{\lambda_{0}}\left(a_{1} \frac{r^{2}}{4}+B \frac{r^{3}}{9}+C \frac{r^{4}}{16}+D \frac{r^{5}}{25}\right)=C_{2}
$$

where $C_{2}$ is a constant. Using the boundary condition (A.4), we find $C_{2}$

$$
C_{2}=U\left(R_{1}\right)+\frac{(m+1) Q_{0}}{\lambda_{0}}\left(a_{1} \frac{R_{1}^{2}}{4}+B \frac{R_{1}^{3}}{9}+C \frac{R_{1}^{4}}{16}+D \frac{R_{1}^{5}}{25}\right)
$$

The substitution of $C_{2}$ into (A.10) gives

$$
U(r)=U\left(R_{1}\right)+\frac{(m+1) Q_{0}}{\lambda_{0}}\left[\frac{a_{1}}{4}\left(R_{1}^{2}-r^{2}\right)+\frac{B}{9}\left(R_{1}^{3}-r^{3}\right)+\frac{C}{16}\left(R_{1}^{4}-r^{4}\right)+\frac{D}{25}\left(R_{1}^{5}-r^{5}\right)\right] .
$$

Finally, by using (A.2), we obtain the required solution of (3.2) in the form (4.6):

$$
T_{g}(r)=\left\{T_{1}^{m+1}+\frac{(m+1) Q_{0}}{\lambda_{0}}\left[\frac{a_{1}}{4}\left(R_{1}^{2}-r^{2}\right)+\frac{B}{9}\left(R_{1}^{3}-r^{3}\right)+\frac{C}{16}\left(R_{1}^{4}-r^{4}\right)+\frac{D}{25}\left(R_{1}^{5}-r^{5}\right)\right]\right\}^{1 /(m+1)} .
$$

\section{Acknowledgments}

This work is partially supported by the National Scientific Fund of Bulgarian Ministry of Education and Science, Grant no. VU-MI-205/2006.

\section{References}

[1] N. K. Vuchkov, K. A. Temelkov, and N. V. Sabotinov, "UV lasing on $\mathrm{Cu}^{+}$in $\mathrm{Ne}-\mathrm{CuBr}$ pulse longitudinal discharge," IEEE Journal of Quantum Electronics, vol. 35, pp. 1799-1804, 1999.

[2] N. K. Vuchkov, K. A. Temelkov, P. V. Zahariev, and N. V. Sabotinov, "Influence of the active zone diameter on the UV ion $\mathrm{Ne}-\mathrm{CuBr}$ laser performance," IEEE Journal of Quantum Electronics, vol. 37, pp. 1538-1546, 2001. 
[3] K. Beev, K. Temelkov, N. Vuchkov, et al., "Optical properties of polymer films for near UV recording," Journal of Optoelectronics and Advanced Materials, vol. 7, pp. 1315-1318, 2005.

[4] M. Ilieva, V. Tsakova, N. K. Vuchkov, K. A. Temelkov, and N. V. Sabotinov, "UV copper ion laser treatment of poly-3,4- ethylenedioxythiophene," Journal of Optoelectronics and Advanced Materials, vol. 9, pp. 303-306, 2007.

[5] N. K. Vuchkov, K. A. Temelkov, and N. V. Sabotinov, "Effect of hydrogen on the average output power of the UV Cu+ ${ }^{+} \mathrm{Ne}-\mathrm{CuBr}$ laser," IEEE Journal of Quantum Electronics, vol. 41, pp. 62-65, 2005.

[6] N. Vuchkov, "High discharge tube resource of the UV Cu${ }^{+} \mathrm{Ne}-\mathrm{CuBr}$ laser and some applications," in New Developments in Lasers \& Electro-Optics Research, W. T. Arkin, Ed., pp. 41-47, Nova Science, Lancaster, UK, 2006.

[7] Yu. G. Gradoboev, Yu. V. Gulyaev, M. A. Kazaryan, et al., "UV radiation source based on a copper vapour laser with acousto-optically controlled spectral and temporal parameters," Quantum Electronics, vol. 34, pp. 1133-1137, 2004.

[8] Y. P. Raizer, M. N. Shneide, and N. A. Yatsenko, Radio-Frequency Capacitive Discharges, CRC Press, New York, NY, USA, 1995.

[9] M. J. Kushner and B. E. Warner, "Large-bore copper-vapor lasers: kinetics and scaling issues," Applied Physics, vol. 54, pp. 2970-2982, 1983.

[10] A. M. Boichenko, G. S. Evtushenko, O. V. Zhdaneev, and S. I. Yakovlenko, "Theoretical analysis of the mechanisms of influence of Hydrogen additions on the emission parameters of a copper vapour laser," Quantum Electronics, vol. 33, pp. 1047-1058, 2003.

[11] B. A. Ghani and M. Hammadi, "Modeling the plasma kinetics mechanisms of CuBr laser with neonhydrogen additives," Optics $\mathcal{E}$ Laser Technology, vol. 38, no. 2, pp. 67-76, 2006.

[12] R. J. Carman, D. J. W. Brown, and J. A. Piper, "A self-consistent model for the discharge kinetics in a high-repetition-rate copper-vapor laser," IEEE Journal of Quantum Electronics, vol. 30, no. 8, pp. 1876-1895, 1994.

[13] R. J. Carman, R. P. Mildren, M. J. Withford, D. J. W. Brown, and J. A. Piper, "Modeling the plasma kinetics in a kinetically enhanced copper vapor laser utilizing $\mathrm{HCl}+\mathrm{H}_{2}$ admixtures," IEEE Journal of Quantum Electronics, vol. 36, no. 4, pp. 438-449, 2000.

[14] B. L. Pan, G. Chen, B. N. Mao, and Z. X. Yao, "Kinetic process of UV Cu ${ }^{+}$laser in Ne-CuBr longitudinal pulsed discharge," Optics Express, vol. 14, no. 19, pp. 8644-8653, 2006.

[15] D. N. Astadjov, N. K. Vuchkov, and N. V. Sabotinov, "Parametric study of the CuBr laser with hidrogen additives," IEEE Journal of Quantum Electronics, vol. 24, pp. 1926-1935, 1988.

[16] K. A. Temelkov, N. K. Vuchkov, B. L. Pan, N. V. Sabotinov, B. Ivanov, and L. Lyutov, "Strontium bromide vapor laser excited by nanosecond pulsed longitudinal discharge," in 14th International School on Quantum Electronics: Laser Physics and Applications, vol. 6604 of Proceedings of SPIE, pp. 15, March 2007.

[17] I. P. Iliev, S. G. Gocheva-Ilieva, and N. V. Sabotinov, "Analytic study of the temperature profile in a copper bromide laser," Quantum Electronics, vol. 38, pp. 338-342, 2008.

[18] S. G. Gocheva-Ilieva, I. P. Iliev, K. A. Temelkov, N. K. Vuchkov, and N. V. Sabotinov, “Analytical model of the temperature in $\mathrm{UV} \mathrm{Cu}^{+} \mathrm{CuBr}$ laser," in Applications of Mathematics in Engineering and Economics, M. D. Todorov, Ed., vol. CP1067, pp. 114-121, American Institute of Physics, Melville, NJ, USA, 2008.

[19] N. K. Vuchkov, D. N. Astadjov, and N. V. Sabotinov, "Influence of the excitation circuits on the CuBr laser performance," IEEE Journal of Quantum Electronics , vol. 30, no. 3, pp. 750-758, 1994.

[20] M. N. Özişik, Heat Transfer. A Basic Approach, McGraw-Hill, Boston, Mass, USA, 1985.

[21] V. A. Milchev, D. Z. Uzunov, V. Y. Jordanov, and D. K. Palov, Heating Technology, Tehnika, Sofia, Bulgaria, 1989.

[22] M. Oprev, Tz. Batov, and D. Uzunov, Heating Technology, Tehnika, Sofia, Bulgaria, 1978.

[23] M. Abramowitz and I. Stegun, Handbook of Mathematical Functions, with Formulas, Graphs, and Mathematical Tables, Dover, New York, NY, USA, 9th edition, 1964.

[24] P. Blau, "Impedance matching and electric field penetration in metal vapour lasers," in Pulsed Metal Vapour Lasers, E. Little and N. V. Sabotinov, Eds., vol. 5 of Nato Science Partnership Sub-Series: 1, pp. 215-220, Kluwer Academic Publishers, Dordrecht, The Netherlands, 1996.

[25] http://reference.wolfram.com/mathematica/guide/Mathematica.html.

[26] L. J. Slater, Generalized Hypergeometric Functions, Cambridge University Press, Cambridge, UK, 1966. 


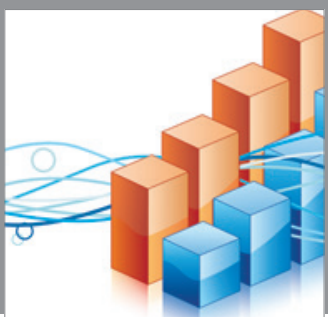

Advances in

Operations Research

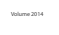

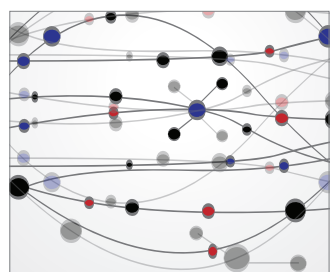

\section{The Scientific} World Journal
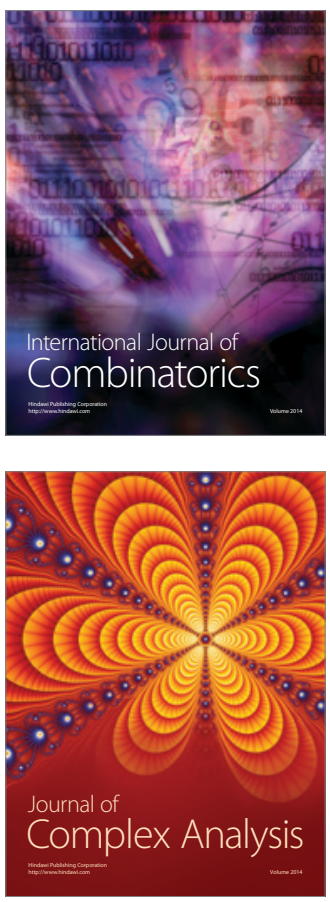

International Journal of

Mathematics and

Mathematical

Sciences
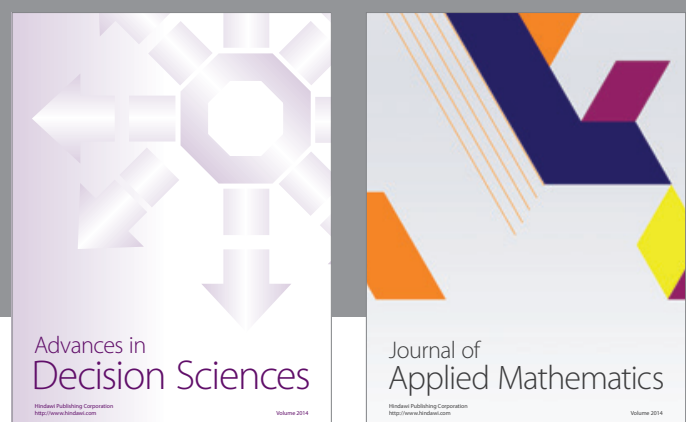

Journal of

Applied Mathematics
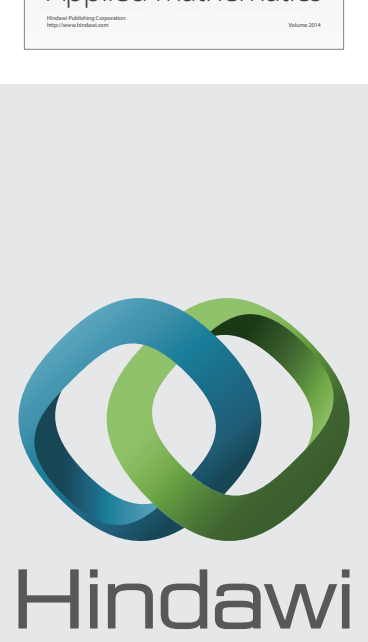

Submit your manuscripts at http://www.hindawi.com
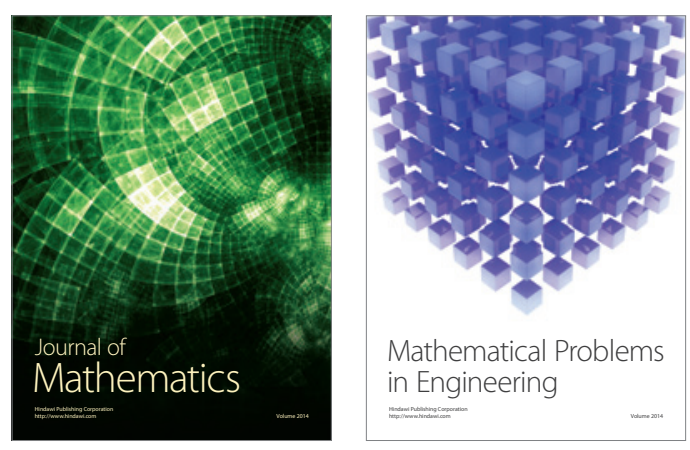

Mathematical Problems in Engineering
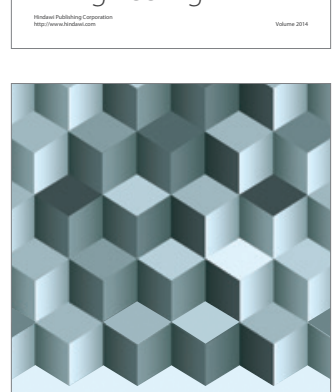

Journal of

Function Spaces
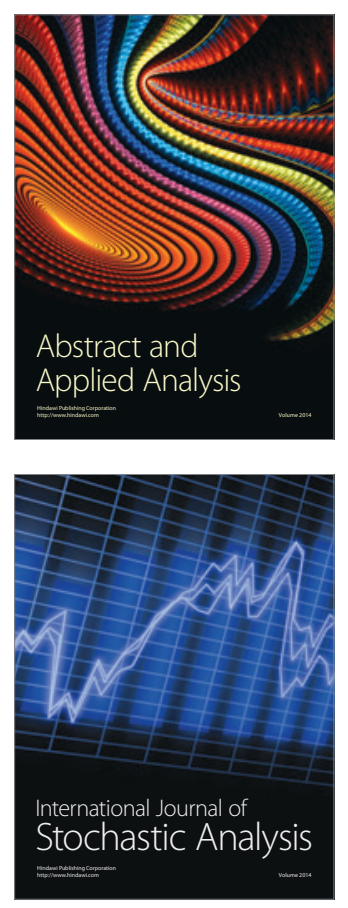

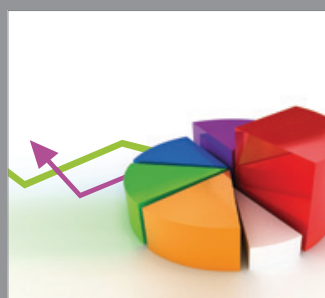

ournal of

Probability and Statistics

Promensencen
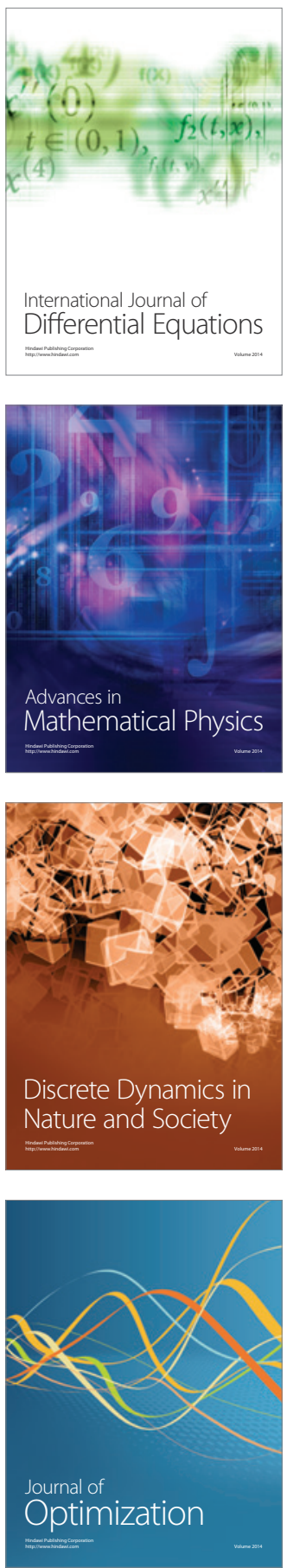